\title{
AN EFFECTIVE ROUTING ALGORITHM FOR V2V COMMUNICATIONS IN URBAN ENVIRONMENT
}

\author{
Tran Van Hung ${ }^{1 *}$ \\ *1 University of Transport and Communications, Hanoi, Vietnam Email: hungtv_ktdt@utc.edu.vn
}

*Corresponding Author: -

Email: hungtv_ktdt@utc.edu.vn

\begin{abstract}
: -
In VANET, the routing is a difficult problem due to unpredictable nodes as vehicles movement and frequent network topology change. Therefore, the most important issue is to predict correctly the future movements of vehicles when selecting intermediate nodes. This paper proposes a Geography Movement Prediction Routing protocol (GMPR) for V2V communication in urban environment that combines vehicle's geography information and movement prediction. Theory analysis and experiments show that the proposed algorithm outperformed the conventional algorithms on the comparisons of packets delivery rate, average end-to-end delay and average number of hops.
\end{abstract}

Keywords: VANET; V2V; 802.11p; GMPR;

\section{(c) $(\$)$}




\section{INTRODUCTION}

VANET-Vehicular Ad hoc NETwork is the core of ITS systems. It was designed to rationalize the operation of vehicles in order to improve road safety. VANETs are self-organized network where packet is delivered by multi-hop fashion. There are two types of communication in this network: V2V - Vehicle to Vehicle and V2I-Vehicle to Infrastructure. The high speed and high mobility of vehicles make it very challenging to establish and maintain a reliable wireless connection, especially in urban environments where communication links frequently be broken by the shielding effect of buildings, leading to a constantly changing network structure and unwanted effects: fading, doppler, transmission delay, etc. So, traditional table-driven routing protocols based on the Bellman-Ford algorithm are no longer fit for such an environment ${ }^{[1]}$.

GPSR $^{[2]}$ is a well-known protocol introducing the greedy forwarding strategy that achieves high performance in free open space, but it is shown that radio obstacles such as buildings degrade the protocol in urban area ${ }^{[3]}$. To address this problem, an intersection-based protocol, GPCR ${ }^{[4]}$ is proposed for urban scenarios. A node at an intersection behaves as a forwarding leader to avoid the local optimum problem caused by buildings ${ }^{[3]}$. GSR ${ }^{[5]}$ is specifically designed for routing in city environment to overcome the disadvantages of GPSR. In GSR ${ }^{[5]}$, source S uses city digital map to find shortest path towards destination D via Dijkstra shortest path algorithm. The LAR ${ }^{[6]}$ is implemented in the Manet, suggests an approach to utilize location information to improve performance of routing protocols by reducing the number of routing messages. Predicting the future topology of the network could play a key role to enhance the ITS as well as the vehicular communications ${ }^{[7]}$.

This paper proposes a routing algorithm for $\mathrm{V} 2 \mathrm{~V}$ communication in an urban environment with a road-based path definition and predictable of the vehicle's future movements. In this algorithm, we use vehicle's geography information and movement prediction. That is a result of the traffic conditions, the urban layout, and the driving requirements to observe the traffic constrains.

The rest of this paper is organized as follows: In Section II, we present a GMPR routing algorithm in urban environment. In Section III, we evaluate algorithm performances through simulations. Finally, Section IV concludes this paper.

\section{GMPR ROUTING ALGORITHM IN URBAN ENVIRONMENT}

\section{$A$. Locating the vehicle via status update message}

In VANET there are two types of messages: common safety messages and event safety messages. Common safety messages is also known as status updates messages, provide information about the state of the vehicle, such as location, speed, direction of travel, etc., are broadcasted with frequency from 2 to 10 times per second. Event safety messages, also known as an emergency alert messages, are intended to alert you to potential traffic accidents such as engine failure, sudden braking, sudden lane shift, etc. Emergency alert messages is only broadcasted when the vehicle was in a dangerous state. In this paper, status update messages are used to locate and predict the moving direction of vehicles. Assume that each vehicle is equipped with a GPS device as well as a DSRC communication device. Therefore, each vehicle can get its geographic coordinates as needed.

Let $S=\{s j \mid j=1,2,3, \ldots, k\}$ be the set of sending nodes, $N j=\{n i \mid i=1,2,3, \ldots, m\}$ is the set of adjacent nodes that in the coverage of the sending node $s j$ respectively. The $s j$ node receives periodically status messages from neighboring nodes, this message contains information about the current node $n i$ in the current time (tcur) such as: position $P_{\text {cur }}^{n_{i}}\left(x_{\text {cur }}^{n_{i}}, y_{\text {cur }}^{n_{i}}\right)$,

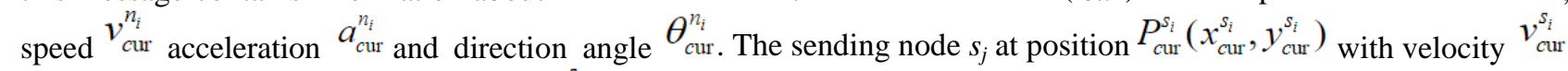
, acceleration $a_{c u r}^{s_{i}}$ and direction angle $\theta_{\text {cur }}^{s_{i}}, n i$ node's position at future time tpred (tpred $\left.=t_{c u r}+\Delta t\right)$ can be calculated:

$$
\begin{aligned}
& x_{\text {pred }}^{n_{i}}=x_{\text {cur }}^{n_{i}}+v_{x}^{n_{i}} \Delta t+0.5 a_{x}^{n_{i}} \Delta t^{2} \\
& y_{\text {pred }}^{n_{i}}=y_{\text {cur }}^{n_{i}}+v_{y}^{n_{i}} \Delta t+0.5 a_{y}^{n_{i}} \Delta t^{2}
\end{aligned}
$$

With:

$$
\left.0 \leq v_{\text {cur }}^{n_{i}}, v_{\text {cur }}^{s_{i}} \leq v_{\max } ; 0 \leq a_{\text {cur }}^{n_{i}}, a_{\text {cur }}^{s_{i}} \leq a_{\max } \text { and } 0 \leq \theta_{\text {cur }}^{n_{i}}, \theta_{\text {cur }}^{s_{i}} \leq a_{\max }\right)
$$

As shown in Figure 1, after sending the status message, the state of $s i$ vehicle will be updated by vehicles $n_{i}, n_{i+1}, n_{i+2}$ and $n i+3$. At the same time, the state of by vehicles $n i, n i+1, n i+2$ and $n i+3$ will be updated by $s i$. In this way each vehicle maintains and updates the list of single- hop adjacent vehicles. The updating cycle of neighboring vehicles list is the status message's broadcasting cycle. 


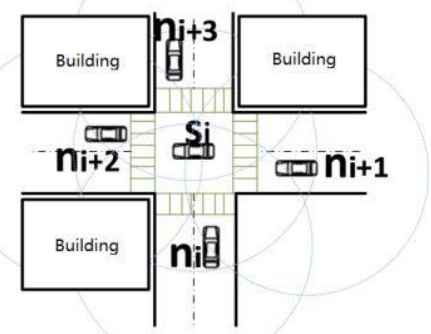

\section{Figure 1. Updated list of nearby vehicles}

\section{B. The next Intersection Selection}

Choosing the next intersection is choosing the next road segment that the message will go through to reach the destination. Proposed algorithm chooses the next intersection based on road segment's actual traffic conditions, including two factors: 1) Vehicle density on the road segment that the message can pass through, and 2) The distance from the intersections can be selected to the destination, which determines the next intersection that is most beneficial for message forwarding. This is an optimal way to find the road in the dynamic state, based on the assessment of both the ability to connect between vehicles through radio communication and the distance to the destination. If the selected road segment has short but vehicles density is too low, so the sending vehicle will not select the next broadcast vehicle, it must keep the message until it can be able to forward. This will increase single-hop delay and reduce routing performance. Relevant road segment information is determined from the digital map database and information from the status message.

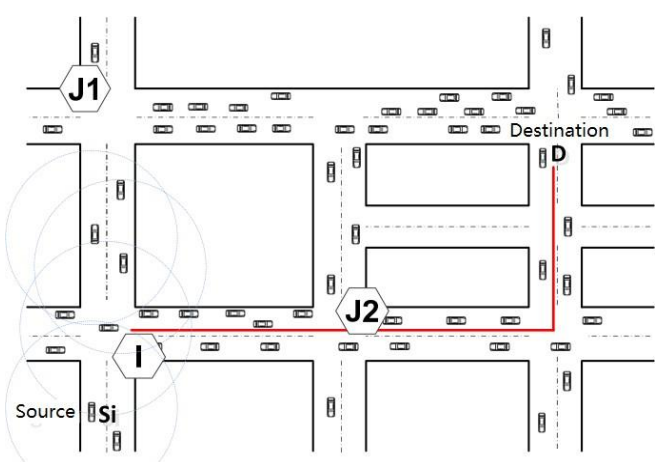

Figure 2. Select the next optimal intersection

As described in Figure 2, sending vehicle $s i$ needs to transmit an event safety messages. The current intersection is $I$, the message can reach destination $D$ via next intersection $J_{I}$ or $J_{2}$. Then, for each vehicle $s i$, it determines a priority function $\mathrm{F}(I J k)$ i for road segment $i$ asfollows:

$$
F\left(I J_{i}\right)_{i}=\alpha \times \eta_{I J_{i}}+\beta \times\left(1-\frac{d_{J_{i} D}}{d_{I D}}\right)
$$

Where $d I D$ is the distance from $I$ to destination $D ; d_{J D}$ is the distance from $J i$ to destination $D ; \eta_{J}$ is the average vehicle density on road segment between two intersection $I$ and $J i ; \alpha, \beta$ is the coefficient of influence of the distance factor and the vehicle density factor, with $\alpha+\beta=1$.

In order to consider the road topology, we use a road graph $G=(V, E)$ composed of intersections as vertices and road segments as edges. On this static graph, the path from source to destination is determined by Dijkstra's shortest path algorithm. The next optimal intersection is chosen that has highest priority function $F(I J k) i$, mean that for each pair of $\alpha$, $\beta$, the road segment is chosen has the highest vehicle density and the shortest distance to the destination.

\section{Message Forwarding}

Once the next intersection has been identified, we can find the groups of vehicles on the road segment that can forward the message to the destination. The next step in the algorithm is to choose which vehicle to forward the message to the next hop is the most beneficial. Vehicles are selected to forward the message based on the priority of distance and have the same moving direction. The selected vehicle has the shortest distance to the next intersection (or destination D if there are no intersections), and is called the "captain" vehicle. In each successive group, the algorithm finds the "captain" vehicle for the next group. The distance from vehicle $n i$ to intersection is calculated as (3), where $P_{J_{\text {opt }}}\left(x_{J_{\text {opt }}}, y_{J_{\text {opt }}}\right)$ is the position of the selectedintersection. 


$$
\left(D_{n_{i}-J_{o p t}}\right)^{2}=\left|x_{\text {pred }}^{n_{i}}-x_{J_{o p t}}\right|^{2}+\left|y_{\text {pred }}^{n_{i}}-y_{J_{o p t}}\right|^{2}
$$

From (1) and (3) we have (4):

$$
D_{n_{i}-J_{\text {opt }}}=\sqrt{\left|x_{c u r}^{n_{i}}+v_{x}^{n_{i}} \Delta t+\frac{1}{2} a_{x}^{n_{i}} \Delta t^{2}\right|^{2}+\left|y_{\text {cur }}^{n_{i}}+v_{y}^{n_{i}} \Delta t+\frac{1}{2} a_{y}^{n_{i}} \Delta t^{2}\right|^{2}}
$$

Selected vehicle $n_{k}$ has a satisfactory distance:

$$
D_{n_{k}-J_{o p t}}=\min \left[D_{n_{i}-J_{o p t}}\right]
$$

Vehicle $n_{k}$ is called the "captain", if vehicle $n_{k}$ does not change the moving direction, the message will be forwarded through other intermediate nodes in the same way. When the next "captain" vehicle is not selected, the current "captain" vehicle will carry the message with "store and forward" technique, keep going until the message reaches to destination.

\section{Vehicle Moving Prediction}

The VANET's network nodes are characterized by their velocity and direction. While transmitting data over the network, the nodes are still moving at high speeds, so without vehicle moving prediction mechanism, messages are sent may not reach the destination because the destination node has moved far away from the location when providing information to the source node. In addition, the relative move between the intermediate node and the destination node is also an important parameter for selecting the path of the message in the routing protocol. By updating the status message, each vehicle always knows its position and position of its nearby intersection. As the vehicle moves in the intersection area, it broadcasts location updates. Receiving this message, neighboring vehicles switch to predictive mode to predict the movement of adjacent vehicles and select the most suitable vehicle to continue forwarding the message.

In Figure 3, when the vehicle $s i$ receives the message from vehicle $s i-1$, vehicle si predicts all the movement of its neighboring vehicles and select the most suitable vehicle $n_{k}$ to continue forwarding the message reach to destination $D$.

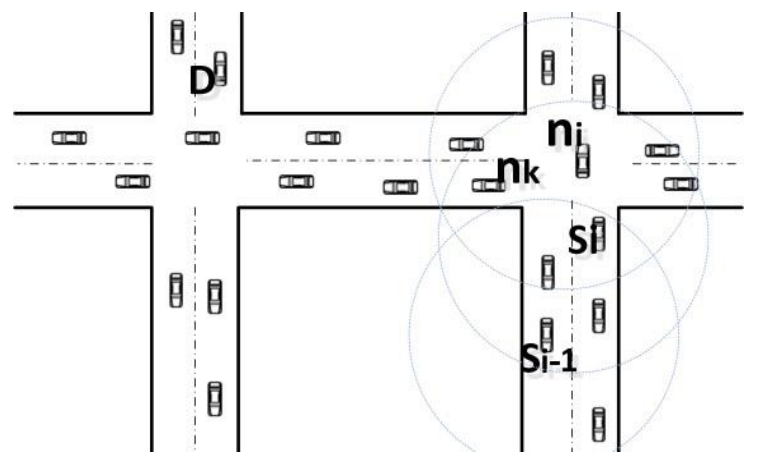

Figure 3. Vehicle $n k$ is choosen by vehicle $n i$

The current position of vehicle $n i$ is $P_{\text {cur }}^{n_{i}}\left(x_{\text {cur }}^{n_{i}}, y_{\text {cur }}^{n_{i}}\right)$, and position of destination vehicle $D$ is $P_{\text {cur }}^{D}\left(x_{\text {cur }}^{D}, y_{\text {cur }}^{D}\right)$. After $\Delta \mathrm{t}$ the new position is determined respectively $P_{\text {pred }}^{n_{i}}\left(x_{\text {pred }}^{n_{i}}, y_{\text {pred }}^{n_{i}}\right) ; P_{\text {pred }}^{D}\left(x_{\text {pred }}^{D}, y_{\text {pred }}^{D}\right)$.

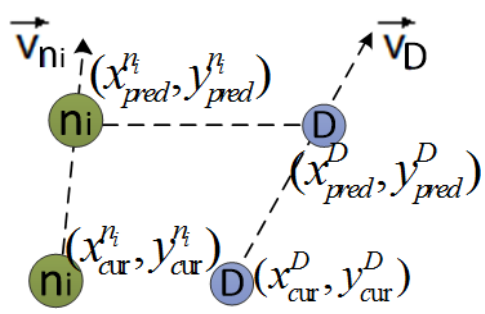

Figure 4. Direction angle between two moving vehicles

Direction angle between two moving vehicles $n_{i} \in N_{j}$ and destination vehicle $\mathrm{D}$ is determined by the formula (6). The vehicle is chosen has a direction angle satisfying formula (7).

$$
\begin{aligned}
\cos \left(\theta_{i}\right) & =\frac{\left(x_{\text {pred }}^{D}-x_{\text {pred }}^{n_{i}}\right)\left(x_{\text {pred }}^{D}-x_{\text {cur }}^{D}\right)+\left(y_{\text {pred }}^{D}-y_{\text {pred }}^{n_{i}}\right)\left(y_{\text {pred }}^{D}-y_{\text {cur }}^{D}\right)}{\sqrt{\left(x_{\text {pred }}^{D}-x_{\text {pred }}^{n_{i}}\right)^{2}+\left(y_{\text {pred }}^{D}-y_{\text {pred }}^{n_{i}}\right)^{2}} \times \sqrt{\left(x_{\text {pred }}^{D}-x_{\text {cur }}^{D}\right)^{2}+\left(y_{\text {pred }}^{D}-y_{\text {cur }}^{D}\right)^{2}}} \\
\theta_{k} & =\text { theta }_{\min }=\min \left\{\theta_{i} \mid \theta_{i}=\left(\overrightarrow{V_{n_{i}}}, \overrightarrow{V_{D}}\right), i=1,2,3, \ldots, m\right\}
\end{aligned}
$$




\section{E. Steps in the GMPR algorithm}

This algorithm performs routing for $\mathrm{V} 2 \mathrm{~V}$ communication in urban environments. The basic steps of the algorithm are as follows:

Step 1: Locate neighboring vehicles within the coverage area of the vehicle by sending a status message, including the current location and prediction location in an interval $\Delta \mathrm{t}$.

Step 2: Based on the distance from sending vehicle to the destination and the density of vehicles on each road segment to select the most beneficial route for the message. This step selects the next optimal intersection.

Step 3: Select intermediate broadcasting vehicles, this step is combination of vehicle movement prediction and finding the shortest paths.

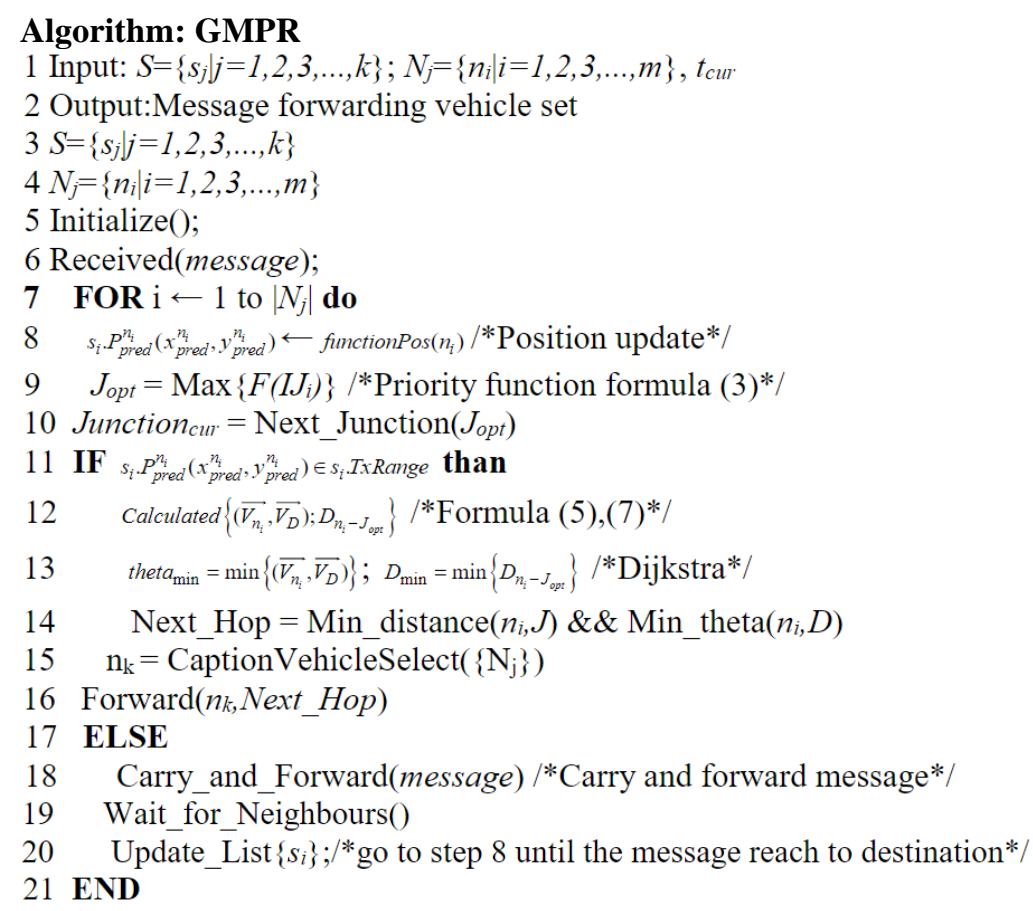

\section{III.SIMULATION AND EVALUATION}

This part simulates the GMPR algorithm for the V2V routing protocol in an urban environment using the NCTUns 6.0 software ${ }^{[9]}$ based on Feroda12. This is a network simulator and emulator software was developed by Taiwan National Chiao Tung University, integrating ITS simulator with IEEE 802.11(p)/1609 standard. Parameters for the simulation are set as in Table 1 .

Table 1. Parameters in simulation

\begin{tabular}{|l|l|l|l|}
\hline Parameters & Value & Parameters & Value \\
\hline Simulation time & $200 \mathrm{~s}$ & MAC protocol & IEEE $802.11(\mathrm{p})$ \\
\hline Number of intersections & 23 & Channel capacity & $2 \mathrm{Mbps}$ \\
\hline Number of road segment & 26 & Message period & $0,2 \mathrm{~s} \sim 0,8 \mathrm{~s}$ \\
\hline Number of vehicles & $50-200$ & Message size & 128 bytes \\
\hline Average speed & $40-60 \mathrm{~km} / \mathrm{h}$ & Area coverage & $250 \mathrm{~m}$ \\
\hline
\end{tabular}

To evaluate the performance of the GMPR algorithm in the paper, we compares the proposed algorithm with a number of routing algorithms already available such as: GPSR ${ }^{[2]}, \mathrm{LAR}^{[6]}, \mathrm{GSR}^{[5]}$. We investigate three performance factors: packet delivery ratio, average end-to- end delay, and average number of forwarding hops. Simulation was simulated 5 times and then took the average of the resulting values.

In the simulation environment, size of the simulation areas is set to $3000 \times 3000 \mathrm{~m}^{2}$. The moving velocities of the vehicles are set to range from $40 \mathrm{~km} / \mathrm{h}$ to $60 \mathrm{~km} / \mathrm{h}$, and the transmission ranges of vehicles are $250 \mathrm{~m}$. The number of total vehicles in our simulations is given as 50, 100, 150, and 200.

Figure 5 gives the packet delivery ratio comparison results of four algorithms with different number of vehicles. As shown in figure 5, when the number of vehicles is small (in case 50 vehicles), proposed algorithm GMPR has slightly poorer performance (the packet delivery ratio) than the GPSR, but outperforms the other three conventional routing algorithms (the LAR, GSR, and GPSR) as the number of vehicles increases. In GMPR algorithm, we investigated the effect of vehicle density on packet routing, when the vehicle density was high enough, the probability of relay success was increased because radio communication was uninterrupted. 


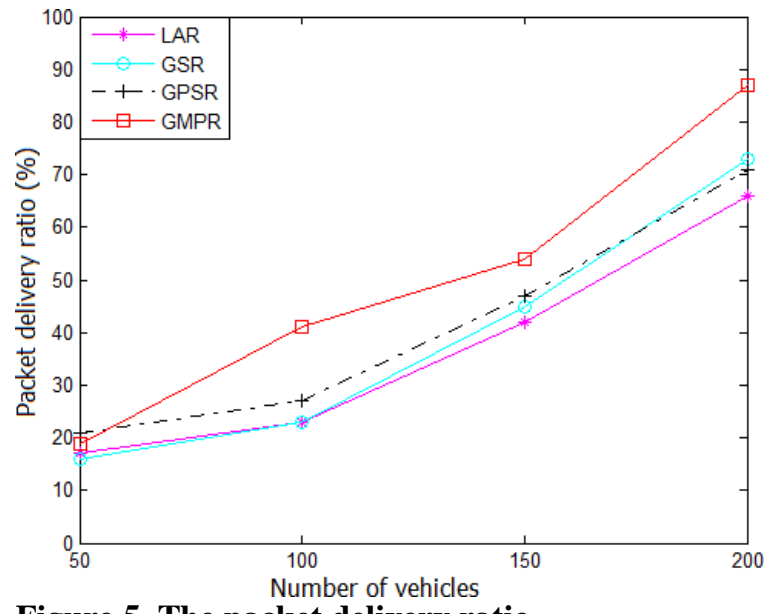

Figure 5. The packet delivery ratio

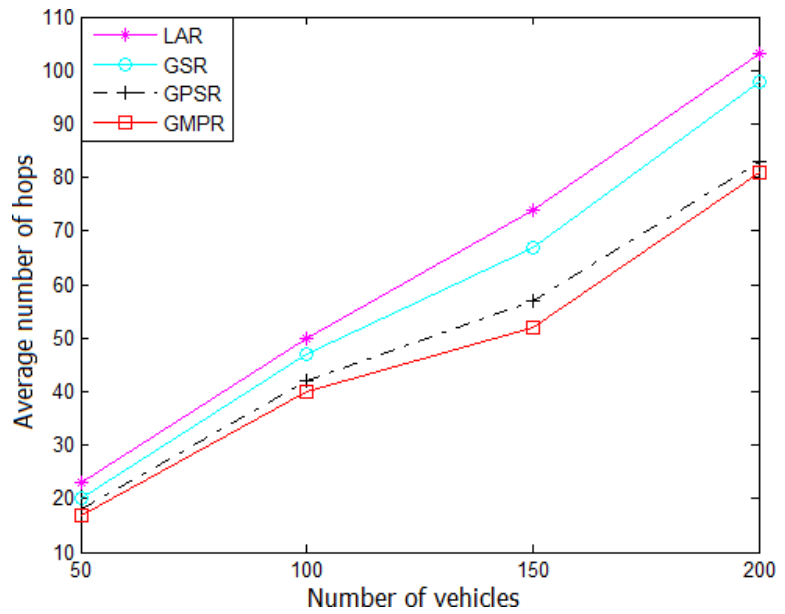

Figure 6. The average number of hops

We investigate the average number of hops required to relay the packet from the source to the destination. The compared methods include the the LAR, GSR, GPSR, and proposed algorithm GMPR. Figure 6 gives the average number of hops and our proposed method GMPR is very close to the optimum number of hops. Because GMPR algorithm combines vehicles location information and vehicles movement prediction, so it always selects the optimal intermediate broadcast node, the other algorithms (LAR, GSR, GPSR) do not have this mechanism.

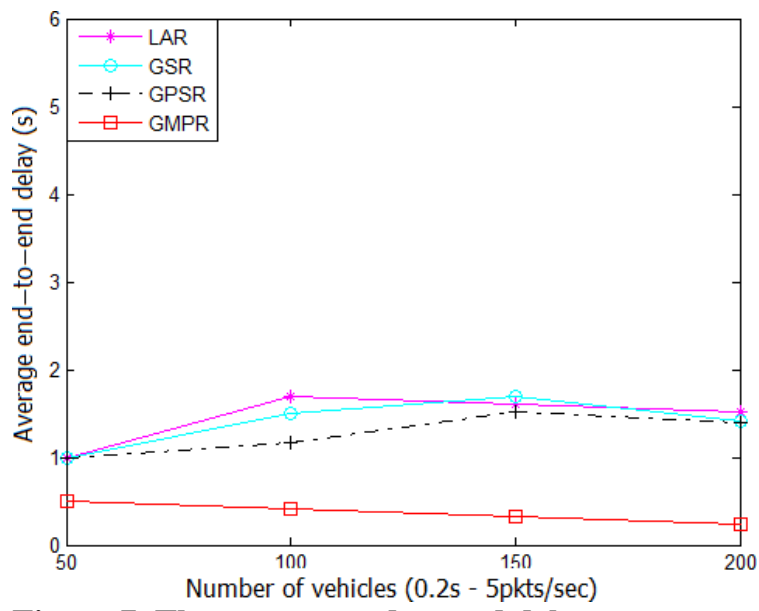

Figure 7. The average end-to-end delay $(5 \mathrm{pkts} / \mathrm{sec})$

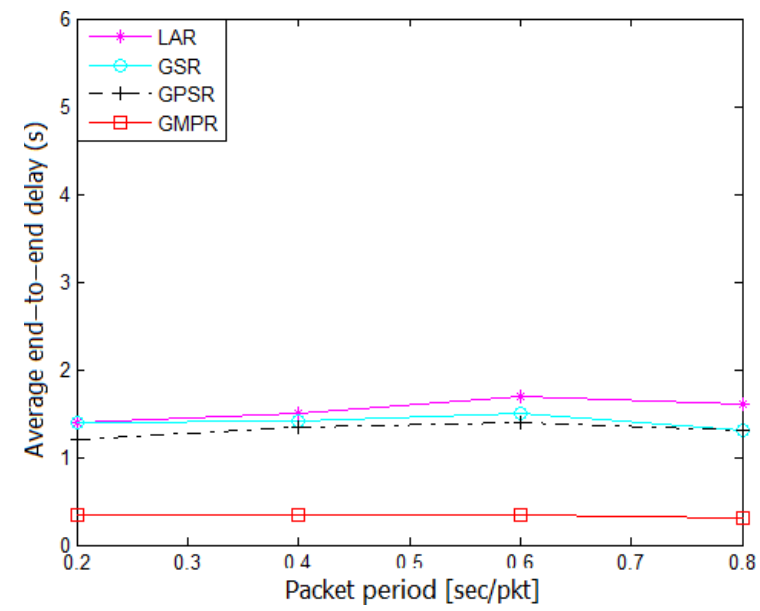

Figure 8. The average end-to-end delay (200 vehicles)

The performance results for the average end-to-end delay comparisons are given in Figure 7 and Figure 8. Our proposed method GMPR also outperformed the other conventional routing algorithms as the number of vehicle nodes varied, which means it uses less time to deliver the packets compared to the other algorithms.

Summarizing the above analysis our proposed GMPR algorithm outperformed the conventional algorithms (LAR, GSR, and GPSR) on the comparisons of packet delivery ratio and average end-to-end delay in the VANETs. The simulation analysis of the number of hops between source and destination comparison also shows that the GMPR algorithm can automatically select the optimal.

\section{IV.CONCLUSION}

In a VANETs environment, directly applying the traditional routing protocols will result in poor performance. Therefore, designing suitable routing protocols for such environments is an emerging area of research. This paper proposes the routing algorithm for $\mathrm{V} 2 \mathrm{~V}$ communication in urban environments, using location information and movement prediction of vehicles. The proposed algorithm has improved the limitations of routing mechanisms based only on location information, improving routing performance. Through theoretical analysis and simulation evaluation, it was shown that the proposed algorithm increased the success rate of the message, reduced the average end-to-end delay time, and reduced the average number of hops when compared with some conventional routing algorithms.

\section{REFERENCES}

[1].Chu-Fu Wang et al, "Nexthop selection mechanism for nodes with heterogeneous transmission range in VANETs", Computer Communication 55, pp. 22-31, 2015

[2].B. Karp and H. T. Kung, "GPSR: Greedy Perimeter Stateless Routing for Wireless Networks", Proc. of ACM MobiCom, 2000, pp. 243-254. 
[3].Sardar M.B, Carlos J.B, Carmen G, "Position-based routing in vehicular networks: A survey", Journal of Network and Computer Applications 36, pp. 685-697, 2013.

[4].C. Lochert, M. Mauve, H. Fussler, and H. Hartenstein, "Geographic Routing in City Scenarios," ACM SIGMOBILE Mobile Computing and Communications Review, vol. 9, no. 1, pp. 69-72, 2005.

[5].Lochert C et al, "Geographic routing in city scenarios", ACM SINGMOBILE Mobile Computing Communication, 9(1):69-72. 2005.

[6].KO Y, VAIDYA N, “Location-Aided Routing in Mobile Ad-hoc Networks”, ACM, IEEE MOBICOM'98. Dallas, pp.66-75.1998

[7].Islam T.A.H, Hossam M.A.F, "Prediction-based protocols for vehicular Ad Hoc Networks: Survey and taxonomy", Computer Networks 130, pp.34-50, 2018

[8].B.L.Souaad, S. Fouzi, B.M. Louiza, "Geographic routing protocols for Vehicular Ad hoc NETworks (VANETs): A survey”, Vehicular Communications 11, pp. 20-31, 2018.

[9].Prof. Shie-Yuan Wang, Chih-Liang Chou, and Chih-Che Lin, "The GUI User Manual for the NCTUns 6.0 Network Simulator and Emulator", Department of Computer Science, National Chiao Tung University, Taiwan.

[10]. Islam T.A.H, Hossam M.A.F, "Prediction-based protocols for vehicular Ad Hoc Networks: Survey and taxonomy", Computer Networks 130, pp. 34-50, 2018. 